\title{
Effects of an educational intervention based on the multi-theory model on promoting the quality of life in postmenopausal women: a protocol
}

\author{
Mohammad Ali Morowatisharifabad ${ }^{1}$, Nooshin Yoshany ${ }^{1,2}$, Narges Bahri ${ }^{3}$, Manoj Sharma $^{4}$, \\ Sara Jambarsang ${ }^{5}$, Zohreh Karimiankakolaki ${ }^{6}$
}

${ }^{1}$ Department of Health Education and Promotion, School of Public Health, Shahid Sadoughi University of Medical Sciences, Yazd, Iran ${ }^{2}$ Social Determinants of Health Research Center, Shahid Sadoughi University of Medical Sciences, Yazd, Iran

${ }^{3}$ Department of Midwifery, Faculty of Medicine, Social Development and Health Promotion Center, Gonabad University of Medical Sciences, Gonabad, Iran

${ }^{4}$ School of Public Health, Jackson State University, Jackson, United States

${ }^{5}$ Department of Bio-statistics and Epidemiology, Shahid Sadoughi University of Medical Sciences, Yazd, Iran

${ }^{6}$ Department of Health, Faculty of Medical Sciences, Shahrekord Branch, Islamic Azad University, Shahrekord, Iran

\begin{abstract}
Introduction: Menopause is associated with symptoms and complications that can affect women's quality of life. Therefore, acceptance of the symptoms and complications can improve quality of life during this period. In this regard, the purpose of this study was to design an educational program based on the multi-theory model (MTM) to deal with complications of the menopausal period and improve the women's quality of life.

Material and methods: In designing this study, four phases are considered. In the first phase, the questionnaire of menopausal symptom acceptance behaviors will be designed based on the MTM using literature review and a panel of experts' viewpoints. The validity and reliability of the questionnaire will be confirmed at this stage. In the second phase, a descriptive study will be conducted by administering the questionnaire designed in the first phase along with the Menopause-Specific Quality of Life questionnaire. The third phase includes the curriculum design based on the findings of the descriptive study, investigations of various studies, and viewpoints of the experts' panel. Therefore, the main components of the intervention will be identified. These components will determine the influential constructs of the MTM according to the descriptive research. Later, the related interventions and messages will be produced and designed from different sources. Intervention strategies will include group discussion, lectures, confidence-building skills, movie screenings, role play, preparation of daily activities booklets for postmenopausal women, and training classes for husbands and children to improve social support for women. The interventions, contents, and messages designed with the presence of health professionals and members of the target community will be pre-tested by examining factors such as audience perception of the message, appropriateness of the education to the audience's literacy and culture, as well as attractiveness, credibility, and acceptance of the materials. Finally, the fourth phase will be the implementation of the pre-test/post-test educational intervention using the intervention and control groups.

Results and conclusions: The present study provides good information about the needs and strategies to enhance the quality of life in postmenopausal women by utilizing a menopausal acceptance training program. Therefore, designing a program to increase the menopausal acceptance in postmenopausal women can be effective in improving the quality of life and reducing the treatment and medical care costs.
\end{abstract}

Key words: menopause, educational intervention, multi-theory model, protocol study.

\section{Introduction}

\section{Background and rationale}

Menopause, as a natural phenomenon and a physiological transition in middle-aged women, is individually, socially, and culturally important. It is associated with complications and consequences that can affect women's quality of life and weaken the good and healthy feeling in them [1]. The mean age of menopause varies in different countries; it was reported as 51, 47.8, and 45.6-47 years in the United States [2], Iran [3], and Turkey [4]. The global average age of menopause 
is 42-58 years with a median of 51.4 years in normal women [3]. Symptoms observed in postmenopausal women include hot flashes, sweating, palpitations, sleep disorders, irritability, lethargy, depressed mood, forgetfulness, decreased libido, depression, vaginal dryness, painful intercourse, urinary symptoms, memory reduction, decreased concentration, restlessness, as well as joint and bone problems. Moreover, during this period, gradual changes are observed in bone metabolism, which increase the frequency of bone fractures [5]. The generally accepted definition for quality of life is the subjective sense of well-being derived from current life experiences. Various factors affect individuals' quality of life [6]. Quality of life is a multidimensional concept that encompasses physical, emotional, and social dimensions of illness and treatment [7]. Menopausal symptoms are closely related to women's quality of life during the menopause transition and can physically, psychologically, and socially change women's quality of life. Health education interventions are one of the alternative strategies for promoting health and coping with menopausal symptoms [8]. Studies have shown that the severity of the menopausal symptoms is associated with low educational levels, low socioeconomic status, race, frequency of deliveries, and high age at menopause onset [9]. Although menopause is one of the natural events in every woman's life span, some of its symptoms are so severe that they affect daily activities and relationships. Moreover, most postmenopausal women do not have adequate information about menopausal symptoms and are not aware of the strategies to control their side effects [10-12]. The first step in promoting the women's compatibility and acceptance of the menopausal changes is to enhance their awareness about the physiological process and the body functions. Awareness about the signs and symptoms of menopause can help women to live a comfortable and happy life. It also alleviates their psychological problems [13].

The MTM, designed by Sharma in 2015, has two important parts: starting behavior change and maintaining the behavior. This model applies the behavioral confidence and changes in the physical environment to initiate behavior changes using participatory dialogue. With regard to maintaining the behavioral change, the MTM uses practice for change, emotional transformation, and changes in the social environment.

The participatory dialogue construct involves discussion about advantages and disadvantages of the behavioral changes in a participatory manner involving mutual communications. The behavioral confidence construct is an embodiment of confidence in health behavior change in the future. The construct of changes in the physical environment refers to changes in the availability, convenience, and readiness of the resources. The emotional transformation construct involves the transformation or transmission of emotions to change the behavior. The construct of practice for change includes constant thinking about changing behavior, making inter-course adjustments, overcoming obstacles, and focusing on healthy behaviors. The construct of changes in the social environment includes the creation of social support in the environment [14].

The trial is based on the Supporting Policy In health with Research: an Intervention Trial (SPIRIT) Action Framework, which is an evaluation over the impact of educational interventions based on the MTM on postmenopausal quality of life. The SPIRIT is a steppedwedge trial that evaluates a complex intervention and uses an objective measure as its primary outcome. It extends previous studies, which evaluated only one strategy [15-17], did not use objective outcome measures $[15,16,18]$, and were unable to include a detailed process evaluation [15-19].

\section{Objectives}

The purpose of presenting this protocol is to investigate the effects of an educational intervention based on the MTM over improving the quality of life among postmenopausal women.

Given the main purpose of this study, several hypotheses were considered according to the viewpoints represented by a panel of reproductive health and health education specialists: the mean scores of participatory dialogue, behavioral confidence, changes in the physical environment, emotional transformation, practice for change, and changes in the social environment constructs will be higher in the intervention than the control group in terms of improving quality of life among the postmenopausal women. Furthermore, mean scores of the postmenopausal women's behavior and quality of life will be higher in the intervention compared to the control group.

\section{Trial design}

In designing this study, four phases are considered. In the first phase, the questionnaire of menopausal symptom acceptance behaviors will be designed based on the MTM using literature review and a panel of experts' viewpoints. The validity and reliability of the questionnaire will be confirmed at this stage. In the second phase, a descriptive study will be conducted by administering the questionnaire designed in the first phase along with the standard MENQOL questionnaire. The third phase includes the curriculum design based on the findings of the descriptive study, investigation of various studies, and viewpoints of the experts' panel. The curriculum will include group discussion, lectures, confidence-building skills, movie screenings, role play, preparation of daily activities booklets for postmeno- 
First phase: Tool making

Second phase: Descriptive study
Designing a tool for identifying the effective factors on the quality of life in postmenopausal women based on the multi-theory model

A descriptive study to identify the status of compliance behaviors with menopause based on the multi-theory model and to evaluate the quality of life in postmenopausal women

Third phase: Designing the educational intervention
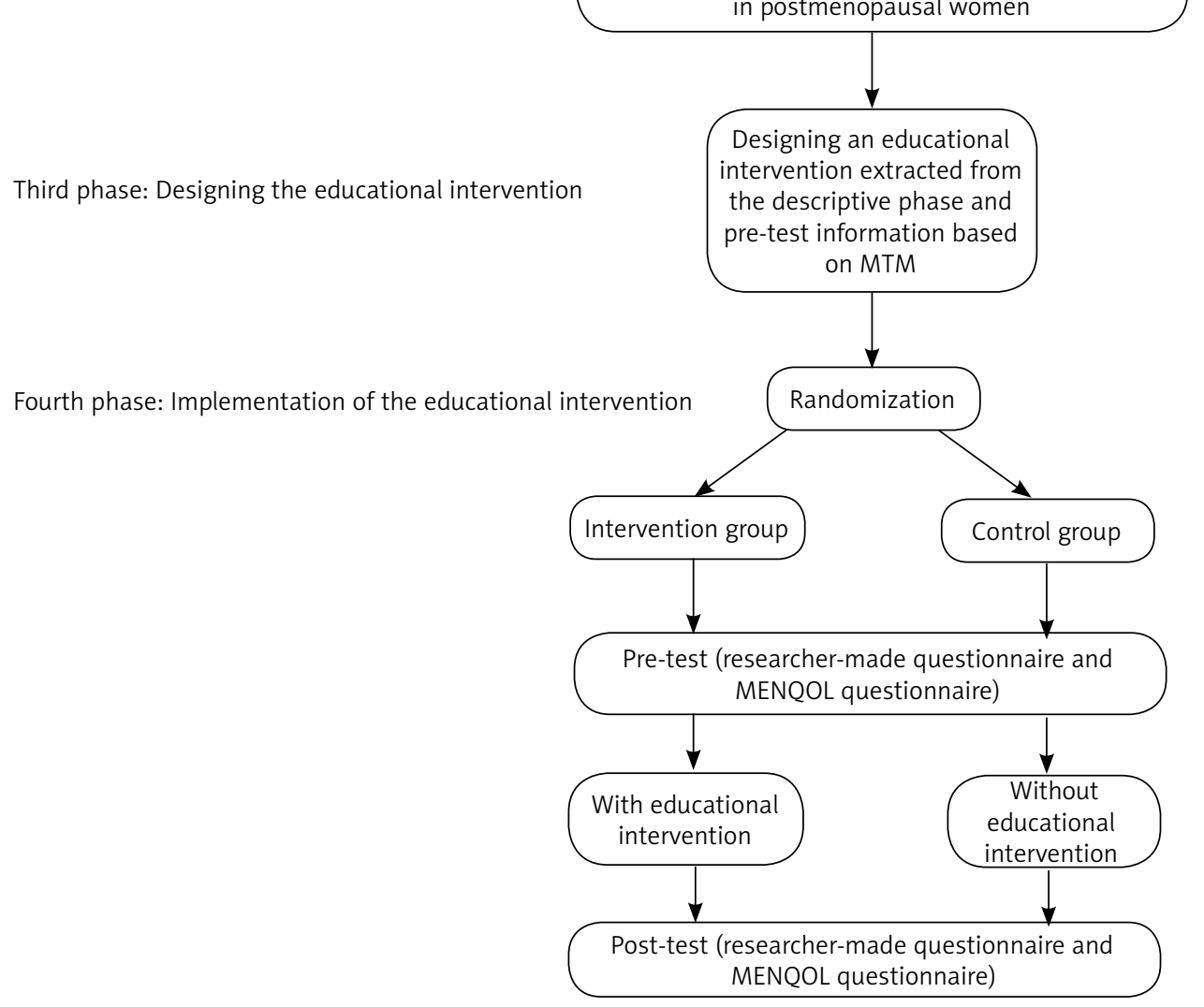

Fig. 1. Flow diagram of thmenopause acceptance behavior trial based on MTM

pausal women, and training classes for husbands and children to improve social support for women. The interventions, contents, and messages designed with the presence of health professionals and members of the target community will be pre-tested by examining factors such as audience perception of the message, appropriateness of the education to the audience's literacy and culture, as well as attractiveness, credibility, and acceptance of the materials. Finally, the fourth phase will be the implementation of the pre-test- post-test educational intervention with intervention and control groups (Fig. 1).

\section{Phase I: Designing the questionnaire of menopausal symptom acceptance behaviors based on the multi-theory model}

At this stage, the literature will be reviewed to select the appropriate model. In this regard, the studies and guidelines on menopause care will be investigated. Moreover, a panel of health education, health promotion, and reproductive health professionals will be interviewed. Then, the questionnaire items will be designed based on the MTM and considering the behavioral acceptance of complications and problems during menopause, which affects the quality of life in postmenopausal women. The behavioral acceptance consists of effective adaptation to hot flashes, night sweats, sleep disorders, mood changes, work-life changes, and social environment changes during the menopause. The questionnaire includes constructs of the participatory dialogue, behavioral confidence, changes in the physical environment, emotional transformation, practice for change, and changes in the social environment. The aim of this questionnaire is to promote quality of life and behavior in postmenopausal women. Validity of the questionnaire will be confirmed by a panel of 15 professionals in health education, health promotion, 
and reproductive health. Moreover, the content validity index and content validity ratio will be obtained to confirm its construct validity. To assess the reliability of the questionnaire, internal consistency will be evaluated by Cronbach's $\alpha$. Consequently, this questionnaire will be used in the pre-test and post-test phases of the intervention.

\section{Phase II: A cross-sectional descriptive study}

This phase will be conducted in health centers of Yazd City and the participants will include the postmenopausal women who referred to these health centers. The inclusion criteria will be having no menses for one year and being in the age range of 45-55 years. The exclusion criteria will include reluctance to continue the study and reluctance to receive the health care services from the health centers. The participants will be randomly selected to complete the questionnaire developed in the first phase and the standard questionnaire of MENQOL. The descriptive phase is necessary to examine the status of menopausal acceptance behaviors and its determinants affecting the quality of life in postmenopausal women based on the MTM. In this phase, the participants' cultural, social, and economic issues are considered to design the educational intervention.

\section{Phase III: Designing the educational intervention}

At this stage, the intervention will be designed and the target audience will be determined based on the literature review, similar studies, and information obtained from the researcher-made questionnaire. The educational program will be based on the information obtained from the descriptive phase. Furthermore, the basic structure of the training intervention package will be designed based on the most important menopausal acceptance behaviors that affect the quality of life in postmenopausal women. Based on the information collected from the pre-test, such as the harmful behaviors in menopause, behavior-changing strategies will be designed to improve the quality of life in postmenopausal women. The educational content will be provided using up-to-date scientific resources (including the Menopause Society website, books, and related articles). Suitability assessment of materials (SAM) technique will be used to evaluate the suitability of the provided material. The SAM provides a percentage score for each medium that falls into one of the three categories: excellent, appropriate, or inappropriate. In this regard, all 22 SAM titles (distributed under six general groups) are evaluated and scored; the excellent, appropriate, or inappropriate options receive two, one, and zero scores, respectively. In the case that the mentioned factor is not applicable for the media the sign (N/A) or
„No items” will be assigned. The total score is calculated by adding the scores and dividing them by the total number of cases. The resulting score will be reported in percent. Scores for excellent, appropriate, and inappropriate media fall in the ranges of $70-100 \%, 40-69 \%$, and $0-39 \%$, respectively. In the case that the medium is inappropriate, the required corrections will be made, and the respective scores will be recalculated [20].

The readability assessment of materials (RAM) technique will be used to evaluate the readability of the media. This technique examines the difficulty associated with reading an educational medium (specialized contents, typographical errors). To reach this target, the educational content will be sent to the faculty members and some target group members. These individuals will be required to comment on the educational contents using a checklist. Finally, the educational content will be revised based on the received feedback [21].

\section{Phase IV: Implementing the educational intervention}

In this phase, a randomized controlled clinical trial will be designed to assess the efficacy of the prepared protocol. Participants will be categorized into two groups randomly. The designed protocol will be implemented for the intervention group, while the control group will receive no intervention. To observe the ethical considerations, the control group members will be provided with all the educational materials after the study.

\section{Material and methods}

\section{Study setting}

Health centers affiliated to Yazd Shahid Sadoughi University of Medical Sciences will be considered for intervention.

\section{Eligibility criterio}

The target participants in this study will include the postmenopausal women who are referred to health care centers to receive the health care services. In the descriptive phase of the study, the sample size will be calculated to fit the regression model according to the questionnaire developed in the previous phase and the number of its unanswered items. Therefore, women in the age range of 45-55 years (as Paszkowski and Skrzypulec-Plinta reported in their study, median age at menopause is 53 years [46-55 years]) [22] who had no menses for one year will enter this phase of the study. In the intervention phase, after designing the questionnaire and conducting a pilot study on a small sample of 
the target group, the sample size will be determined. As a result, women who had no menses for one year, are in the age range of 45-55 years, had normal menopause, are willing to participate in the study, and have no disease, disability, or conscious disorder will enter the interventional research.

\section{Interventions}

The method of instruction will be selected according to the content type and audience accessibility, and will include group discussion, lectures, confidence-building skills, movie screenings, role play, preparation of daily activities booklets for postmenopausal women, and training classes for husbands and children. The research team will finalize these educational methods according to their priorities. The types of educational messages are listed in Table 1.

\section{Intervention strategies related to behovior initiation (group discussion, lecture, confidence-building workshop, pamphlet preparation)}

In order to initiate the behavioral changes and acceptance of the menopausal complications, small group discussion sessions will be held consisting of 8-12 postmenopausal women and two facilitators and educators according to the participatory dialogue structure. In these sessions, the advantages and disadvantages of change will be discussed. Behavior and acceptance of menopausal complications will be addressed. At the end of the discussion, complications of menopause and their reduction strategies will be presented in the speech format. To improve the behavioral confidence, a workshop will be held with the participation of a psy- chiatrist or a psychologist to teach self-esteem and coping skills. Women will practice self-confidence skills in this workshop. Regarding construct of the physical environment changes, a meeting will be held on the changes that can easily help them to feel better in their environment. Furthermore, the participants will be provided with a pamphlet about the laws and policies, sports facilities, as well as health, care, and nutrition services that exist in their community. The individuals will be asked to voice their opinions and the researchers will consult relevant agencies in the case that lack of facilities was reported by participants.

\section{Intervention strategies related to tracking and maintaining behavior (preparing educational videos, role playing, preparing diary of menopause women's activities, teaching spouses and children to increase the social support for postmenopausal women)}

In order to maintain the adopted behaviors and to have a better acceptance of menopause, an educational movie will be produced based on the emotional changes. This movie aims to portray postmenopausal women who had many complications at the onset of the menopause and were able to overcome the complications by observing the principles. At the end of the movie, the participants will be asked to arouse others' emotions by playing their roles. Considering the construct of practice for change, people will be provided with some notebooks. They are supposed to specify the type and duration of the expected activities and behaviors on a daily basis. This helps people to think regularly about the target activities on a daily basis and strengthens their self-control. The session on the construct of change in the social environment will be held for the spouses and

Table 1. Types of strategies and messages presented in the educational intervention package based on the literature review and the panel of experts

\begin{tabular}{ll}
\hline Types of messages & \multicolumn{1}{c}{ Strategies } \\
\hline Educational messages & $\begin{array}{l}\text { Contents include menopause, its symptoms, menopausal acceptance behaviors, harmful behaviors } \\
\text { in menopause, strategies to change behavior during menopause, and improving quality of life. This } \\
\text { part will be conducted in lecture format (e.g., walking about 30 minutes per day reduces hot flashes) }\end{array}$ \\
\hline $\begin{array}{ll}\text { Participatory dialogue } \\
\text { messages }\end{array}$ & $\begin{array}{l}\text { The topics are provided through group discussion as well as question and answer (e.g., discussing } \\
\text { the advantages and disadvantages of exercise for women) }\end{array}$ \\
\hline $\begin{array}{l}\text { Behavioral confidence } \\
\text { promoting messages }\end{array}$ & $\begin{array}{l}\text { Breaking complex behaviors into simple steps and holding a workshop on increasing self-confidence } \\
\text { skills (e.g. start to exercise step-by-step) }\end{array}$ \\
\hline $\begin{array}{l}\text { Change messages in the } \\
\text { physical environment }\end{array}$ & $\begin{array}{l}\text { Educating people about environmental amenities and accessibilities (e.g., providing a pamphlet to } \\
\text { introduce community-based sports facilities) }\end{array}$ \\
\hline $\begin{array}{l}\text { Motivational messages and } \\
\text { emotional transformation }\end{array}$ & $\begin{array}{l}\text { Including film screenings and role plays (e.g. playing the role of a woman who was able to reduce } \\
\text { her hot flashes by regular exercises) }\end{array}$ \\
\hline $\begin{array}{l}\text { Practice for action messages } \\
\text { Functional messaging }\end{array}$ & Teaching a daily activity log \\
$\begin{array}{l}\text { and changing the social } \\
\text { environment }\end{array}$ & \\
\hline
\end{tabular}


children of the postmenopausal women. In this regard, a health education specialist and a reproductive health specialist will talk about menopausal change, problems of this period, and the supportive role of others. In fact, the spouse and children will be involved in this intervention as "training assistants".

\section{Sample size}

In the descriptive phase of the study, the sample size will be calculated to fit the regression model based on the number of questions left unanswered in the questionnaire prepared in the previous phase. In the interventional phase, sample size has been calculated utilizing $G^{\star}$ Power with a power of 0.80 , and the $\alpha$ of 0.05 that yields a sample size of 20 to detect an effect size of 0.30 . The sampling requirement will be increased by $20 \%$ to 24 participants per group to compensate for any attrition.

\section{Recruitment}

The intervention phase will be carried out as a randomized controlled trial in the intervention and control groups on postmenopausal women who referred to health centers in Yazd City. The participants will be selected using multistage random stratified sampling. In the first stage, health centers of Yazd will be divided into three categories (poor, moderate, and good) with regard to the residents' socioeconomic status. Later, two centers will be selected randomly from each category, which results in six health care centers; three centers will be considered as the intervention group and three centers will be taken as the control group.

\section{Blinding}

The staff and investigators involved in delivering aspects of the intervention, by necessity, are not blind to allocation. However, all other investigators and participants are blind to allocation.

\section{Data collection}

The data collection tools will be a researcher-made questionnaire on menopausal acceptance based on the MTM that includes participatory dialogue, behavioral confidence, changes in the physical environment, emotional transformation, practice for change, and changes in the social environment constructs. The validity and reliability of this questionnaire will be confirmed during the first phase. To collect data on women's quality of life, the MENQOL questionnaire will be used, which includes four dimensions: vasomotor (3 questions), psychosocial (7 questions), physical (16 questions), and sexual (3 questions). The reliability of this questionnaire was confirmed by Abbaszadeh et al. with Cronbach's $\alpha$ coefficient of 0.84 [23]. Furthermore, the content and visual validity of the questionnaire and educational program will be measured by receiving comments from the panel of experts. The panel of experts will include eight professionals in health education, one expert in the health promotion field, six professionals in reproductive health, and one expert in the field of psychology.

\section{Statistical methods}

We will apply the $\chi^{2}$ test, independent $t$-test, and paired $t$-test to analyze our data using SPSS version 21 .

\section{Results}

\section{Multi-theory model constructs}

Mean scores of the participatory dialogue, behavioral confidence, changes in the physical environment, emotional transformation, practice for change, and changes in the social environment constructs will be calculated at pre-test and post-test with regard to promoting quality of life and behaviors of the postmenopausal women.

\section{Quality of life in postmenopausal women}

The mean score of quality of life in postmenopausal women will be obtained using the standard questionnaire of MENQOL at the pre-test and post-test.

\section{Participont timeline}

The detailed participant timeline has been described in Table 2.

\section{Discussion and conclusions}

Several studies have investigated the impact of menopausal acceptance training programs on the quality of life in postmenopausal women. Robak-Chołubek et al. showed that the menopause period has a major impact on the psychological and social well-being of women. The degree of difficulty in experiencing the transition from menopause is also of great importance. Women who have better acceptance with menopause experience fewer problems in their family life and report negative sexual experiences to a lesser degree [24]. Im et al. emphasized that health care providers should develop programs to manage menopausal symptoms with regard to different ethnicities [25]. Caltabiano et al. concluded that conflicting factors were important for 
Table 2. Standard protocol items: recommendation for interventional trials (spirit) diagram of assessments at enrolment, allocation, 2-weekly sessions, post-intervention, immediately and 3-month time interval. Participatory dialogue, behavioral confidence, changes in physical environment, emotional transformation, practice for change, change in social environment for MTM scale, and quality of life for MENQOL scale

\begin{tabular}{|c|c|c|c|c|c|c|}
\hline \multirow[t]{3}{*}{ Item } & \multicolumn{6}{|c|}{ Study period (weeks) } \\
\hline & \multirow{2}{*}{$\begin{array}{c}\text { Enrolment } \\
-8\end{array}$} & \multirow{2}{*}{$\begin{array}{c}\text { Allocation } \\
-2\end{array}$} & \multicolumn{2}{|c|}{ Intervention period } & \multicolumn{2}{|c|}{ Post-intervention } \\
\hline & & & 0 & $+3,+6,+9$ & +9 & +21 \\
\hline Time interval & $-t_{1}$ & 0 & $\mathrm{t}_{1}$ & $t_{2}$ & $\mathrm{t}_{3}$ & $\mathrm{t}_{4}$ \\
\hline \multicolumn{7}{|l|}{ Enrolment } \\
\hline Eligibility screen & $x$ & & & & & \\
\hline Informed consent & $x$ & & & & & \\
\hline Baseline measures & $x$ & & & & & \\
\hline Allocation & & $x$ & & & & \\
\hline \multicolumn{7}{|l|}{ Interventions } \\
\hline Intervention group & & & & $\rightarrow$ & & \\
\hline Control group & & & & $\longrightarrow$ & & \\
\hline \multicolumn{7}{|l|}{ Assessments } \\
\hline Participatory dialogue & & $x$ & & & $x$ & $x$ \\
\hline Behavioral confidence & & $x$ & & & $x$ & $x$ \\
\hline $\begin{array}{l}\text { Changes in physical } \\
\text { environment }\end{array}$ & & $x$ & & & $x$ & $x$ \\
\hline Emotional transformation & & $x$ & & & $x$ & $x$ \\
\hline Practice for change & & $x$ & & & $x$ & $x$ \\
\hline $\begin{array}{l}\text { Change in social } \\
\text { environment }\end{array}$ & & $x$ & & & $x$ & $x$ \\
\hline Quality of life & & $x$ & & & $x$ & $x$ \\
\hline
\end{tabular}

experiencing menopausal and adapting to middle age transition. Furthermore, psychologists and specialists in menopausal clinics are required to promote senses of optimism and integration in postmenopausal women to facilitate their acceptance with this important period of life [26].

Although menopause is one of the natural events in every woman's life span, some of its symptoms are so severe that they affect daily activities and communication. Moreover, most postmenopausal women do not have adequate information about menopausal symptoms and are not aware of the strategies to control their side effects [10-12]. Menopausal symptoms are closely associated with women's quality of life during the menopause and can change women's quality of life physically, psychologically, and socially [8]. In a study by Yazdkhasti et al., health education intervention strategies were reported as one of the alternative strategies for improving women's attitudes and helping them to cope with menopausal symptoms [27].

The present study provides good information on the needs and strategies to enhance the quality of life in postmenopausal women by implementing an educational program over acceptance of menopausal symptoms. Therefore, designing a program that can increase the acceptance in postmenopausal women improves the women's quality of life and can be effective in re- ducing treatment and medical care costs. In the present study, spouses and children of postmenopausal women will be considered as "training assistants" in improving social support for women. In the same vein, Karimiankakolaki et al. used the "training assistant" technique in an intervention program to support pregnant women living with smoker men [28]. The current intervention program will have the potential to serve as a guide for health care providers to improve taking care of postmenopausal women in health care settings. The strategies outlined in this program are cost-effective and can improve the quality of life in postmenopausal women.

\section{Acknowledgements}

This paper was extracted from a health education and promotion Ph.D. thesis. The authors would like to thank Shahid Sadoughi University of Medical Sciences for supporting this research.

The research protocol was funded by Shahid Sadoughi University of Medical Sciences, Yazd, Iran.

\section{Disclosure}

The authors report no conflict of interest. 


\section{References}

1. Sheikhan Z, Ozgoli G, Azar M, et al. Prevalence of sexual violence among infertile women. Adv Nurs Midwifery 2014; 23: 5836.

2. Perry S, Bobak I. Maternity \& Womens Health Care. Mosby Company, London 2000

3. Norozi E, Mostafavi F, Hasanzadeh A, et al. Factors related with quality of life among postmenopausal women in Isfahan, Iran, based on behavioral analysis phase of precede model. Health Syst Res 2011; 7: 267-277.

4. Karaçam Z, Şeker SE. Factors associated with menopausal symptoms and their relationship with the quality of life among Turkish women. Maturitas 2007; 58: 75-82.

5. Krajewska-Ferishah K, Krajewska-Kułak E, Terlikowski S, et al. Analysis of quality of life of women in menopause period in Poland, Greece, Belarus and Belgium using MRS Scale. A multicenter study. Adv Med Sci-Poland 2010; 55: 191-195.

6. Froberg DG, Kane RL. Methodology for measuring health-state preferences - II: Scaling methods. J Clin Epidemiol 1989; 42: 459-471.

7. Pulman A. A patient centred framework for improving LTC quality of life through Web 2.0 technology. Health Informatics J 2010; 16: 15-23.

8. Rotem M, Kushnir T, Levine R, Ehrenfeld M. A Psycho-Educational Program for improving women's attitudes and coping with menopause symptoms. J Obstet Gynecol Neonatal Nurs 2005; 34: 233-240.

9. Friedman SH, Sajatovic M, Schuermeyer IN, et al. Menopause-related quality of life in chronically mentally ill women. Int J Psychiatry Med 2005; 35: 259-271.

10. Chedraui P, Blümel JE, Baron G, et al. Impaired quality of life among middle aged women: a multicentre Latin American study. Maturitas 2008; 61: 323-329.

11. Lu J, Liu J, Eden J. The experience of menopausal symptoms by Arabic women in Sydney. Climacteric 2007; 10: 72-79.

12. Peeyananjarassri K, Cheewadhanaraks S, Hubbard M, et al. Menopausal symptoms in a hospital-based sample of women in southern Thailand. Climacteric 2006; 9: 23-29.

13. Hassan Zadeh GR, Yekkezare S, Vaziri S, et al. Knowledge of women about menopause in Qazvin. J Qazvin Univ Med Sci 2003; 7: 21-25.

14. Sharma M. Multi-theory model (MTM) for health behavior change. WebmedCentral Behaviour 2015; 6: WMC004982.

15. Dobbins M, Cockerill R, Barnsley J, Ciliska D. Factors of the innovation, organization, environment, and individual that predict the influence five systematic reviews had on public health decisions. Int J Technol Assess Health Care 2001; 17: 467-478.

16. Kothari A, Birch S, Charles C. "Interaction" and research utilisation in health policies and programs: does it work? Health Policy 2005; 71 : 117-125.

17. Taylor RS, Reeves BC, Ewings PE, Taylor RJ. Critical appraisal skills training for health care professionals: a randomized controlled trial [ISRCTN46272378]. BMC Medical Education 2004; 4: 30.

18. Denis J-L, Lomas J, Stipich N. Creating receptor capacity for research in the health system: the Executive Training for Research Application (EXTRA) program in Canada. SAGE Publications Sage UK, London, England 2008.

19. Dobbins M, Hanna SE, Ciliska D, et al. A randomized controlled trial evaluating the impact of knowledge translation and exchange strategies. Implement Sci 2009; 4: 61.

20. Hoffmann T, Ladner Y. Assessing the suitability of written stroke materials: An evaluation of the interrater reliability of the suitability assessment of materials (SAM) checklist. Top Stroke Rehabil 2012; 19: 417-422.

21. Rhee RL, Von Feldt JM, Schumacher HR, Merkel PA. Readability and suitability assessment of patient education materials in rheumatic diseases. Arthritis Care Res (Hoboken) 2013; 65: 1702-1706.

22. Paszkowski T, Skrzypulec-Plinta V. Assessment of quality of life in women using Femelis Meno. Prz Menopauzalny 2018; 17: 77-85.

23. Abbaszadeh $M$. Validity and reliability in qualitative researches. J Appl Sociol 2012; 23: 19-34.

24. Robak-Cholubek D, Wdowiak A, Makara-Studzinska M, Korczynska E. Perception and degree of acceptance of menopause-related changes in various spheres of life by postmenopausal women. Ann Agric Environ Med 2014; 21: 666-669.
25. Im E-O, Ko Y, Hwang H, Chee W. "Symptom-Specific or Holistic": Menopausal Symptom Management. Health Care Women Int 2012; 33: 575 592.

26. Caltabiano M, Holzheimer M. Dispositional factors, coping and adaptation during menopause. Climacteric 1999; 2: 21-28.

27. Yazdkhasti M, Keshavarz M, Khoei EM, et al. The effect of support group method on quality of life in post-menopausal women. Iran J Public Health 2012; 41: 78-84.

28. Karimiankakolaki Z, Mahmoodabad SSM, Kazemi A, Fallahzadeh H. Designing an educational intervention on second-hand smoke in smoker men on the exposure of pregnant wives: a protocol for a randomized controlled trial. Reprod Health 2019; 16: 11. 